\title{
Les diodes électroluminescentes et le risque rétinien dû à la lumière bleue
}

\author{
Christophe MARTINSONS \\ Centre scientifique et technique du bâtiment \\ christophe.martinsons@cstb.fr
}

Cet article dresse l'état des connaissances sur la toxicité potentielle sur la rétine de la lumière émise par les diodes électroluminescentes (LED). En raison de leur forte luminance et de leur spectre d'émission comprenant une raie dont le maximum est situé dans le bleu, le risque rétinien potentiel dit de « lumière bleue » a été identifié et étudié depuis plusieurs années. Plusieurs expertises indépendantes menées par des agences de sécurité sanitaire ont montré que le risque présenté par les LED en tant que sources d'éclairage général est faible, mais ne peut être négligé dans certains usages ou pour certaines populations sensibles, étant donné la croissance rapide de l'éclairage à LED et l'augmentation régulière des flux lumineux des composants utilisés.

\section{Les diodes électroluminescentes ef leurs caractéristiques optiques}

Les sources d'éclairage traditionnelles telles que la lampe à incandescence ou la lampe fluorescente compacte sont progressivement remplacées par des dispositifs intégrant des diodes électroluminescentes (LED). L'éclairage à semi-conducteur, dit SSL pour solid-state lighting en anglais, présente un certain nombre d'avantages comme une durée de vie plus longue, une consommation énergétique moindre et des impacts environnementaux généralement plus faibles [1]. La figure 1 montre quelques exemples de LED et de dispositifs d'éclairage utilisant des LED. La Commission européenne, suivant l'exemple de plusieurs gouvernements de par le monde, a programmé de manière réglementaire l'interdiction progressive des lampes les plus énergivores au profit de nouvelles technologies d'éclairage plus efficaces, au rang desquelles les LED apparaissent comme étant incontestablement dominantes. Les acteurs industriels de l'éclairage prédisent d'ailleurs que plus de $90 \%$ de toutes les sources d'éclairage dans le monde seront basées sur des LED d'ici à 2020.

Comme toutes les nouvelles technologies émergentes, les produits d'éclairage
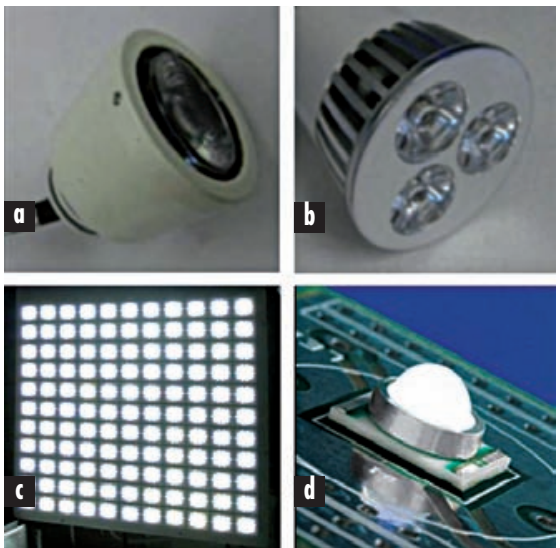

Figure 1. Photographies de plusieurs dispositifs d'éclairage LED. (a) Luminaire directionnel (spot) utilisant une LED. (b) Lampe utilisant trois LED et utilisable en remplacement d'une lampe à incandescence. (c) Luminaire d'éclairage extérieur de forte puissance et utilisant 121 modules de LED. (d) Composant LED typique, utilisé dans de nombreux dispositifs d'éclairage. Ce type de LED a une puissance électrique d'environ $1 \mathrm{~W}$ et génère un flux lumineux supérieur à $100 \mathrm{~lm}$.

utilisant des LED doivent se révéler aussi sûrs que les produits qu'ils prétendent supplanter. De plus, les propriétés uniques des LED comme leur compacité et leurs propriétés colorimétriques ont généré beaucoup de nouvelles applications et de nouveaux usages de l'éclairage qui n'étaient pas envisageables avec les anciennes technologies. À titre d'exemple, on peut citer certains jouets et certains vêtements qui incorporent des LED. Les aspects sécuritaires de ces produits à LED doivent être évalués en considérant les interactions possibles avec le corps humain non seulement selon les usages bien connus mais aussi selon les nouvelles utilisations qui en sont faites.

Les effets néfastes des rayonnements optiques sur la peau et les yeux font partie du domaine de la sécurité photobiologique. Les LED utilisées à I'heure actuelle dans les applications d'éclairage ont l'avantage d'émettre des quantités extrêmement faibles de rayonnements ultraviolet et infrarouge. Le seul risque photobiologique à considérer dans le cas des LED est celui lié à la lumière visible et en particulier aux faibles longueurs d'onde, c'est-àdire la partie bleue et violette du spectre.

Plusieurs agences de sécurité sanitaire comme I'ANSES [2] et le SCENIHR [3] ont récemment fait le point sur la littérature scientifique relative au risque photobiologique présenté par les LED. Deux caractéristiques principales ont attiré l'attention des experts de ces agences:

- Les LED sont de très petites sources de lumière qui peuvent être éblouissantes. 


\begin{tabular}{|c|c|}
\hline Source & Ordre de grandeur de la luminance \\
\hline Soleil, vu au travers de l'atmosphère terrestre & $\begin{array}{l}\text { Plusieurs milliards de } \mathrm{cd} / \mathrm{m}^{2} \\
\text { au milieu de la journée en France }\end{array}$ \\
\hline Ciel clair & Quelques milliers de $\mathrm{cd} / \mathrm{m}^{2}$ \\
\hline Filament d'une lampe à incandescence & Un million de $\mathrm{cd} / \mathrm{m}^{2}$ \\
\hline Enveloppe d'une lampe à incandescence dépolie & 10000 à $50000 \mathrm{~cd} / \mathrm{m}^{2}$ \\
\hline Tube fluorescent et lampe fluocompacte & 5000 à $10000 \mathrm{~cd} / \mathrm{m}^{2}$ \\
\hline $\begin{array}{l}\text { LED blanche alimentée avec une puissance } \\
\text { électrique de } 1 \mathrm{~W} \text { et délivrant un flux lumineux } \\
\text { typique de } 100 \mathrm{~lm}\end{array}$ & Entre 10 et 20 millions $\mathrm{de} \mathrm{cd} / \mathrm{m}^{2}$ \\
\hline $\begin{array}{l}\text { LED blanche utilisée pour l'éclairage automobile } \\
\text { de jour }\end{array}$ & 60 millions de $\mathrm{cd} / \mathrm{m}^{2}$ \\
\hline
\end{tabular}

Tableau 1. Ordres de grandeur de la luminance de différentes sources de lumière.

Elles possèdent une luminance très élevée (tableau 1) qui engendre un niveau d'éclairement important de la rétine.

- La grande majorité des LED blanches sont basées sur une puce émettant de la lumière bleue ou violette associée à des couches de matériaux fluorescents (luminophores) pour produire des rayonnements de plus grandes longueurs d'onde. Par conséquent, le spectre d'émission de ces LED blanches comporte une raie primaire relativement étroite (environ $20 \mathrm{~nm}$ FWMH) et une zone secondaire plus large correspondant à la désexcitation des luminophores dans les parties jaune, orange et rouge du spectre (figure 2). Les deux zones sont séparées par une région de très faible émission située dans la zone vert-bleu du spectre, typiquement vers $480 \mathrm{~nm}$.

\section{Les risques rétiniens liés à la lumière bleue}

Dans le domaine visible, des niveaux d'éclairement trop importants sur la rétine peuvent induire des lésions thermiques et photochimiques. Les lésions thermiques résultent d'une exposition aigue à une source de lumière intense, de manière

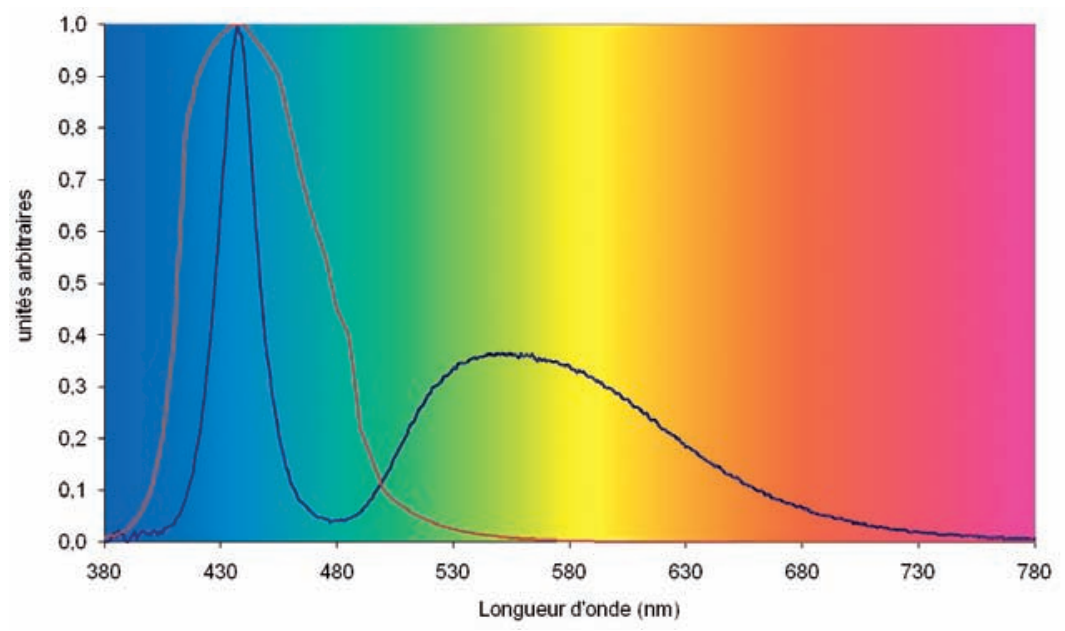

Figure 2. La courbe bleue représente le spectre d'émission typique d'une LED blanche. Dans cet exemple, le pic bleu atteint son maximum vers $435 \mathrm{~nm}$. Il correspond à l'émission de la jonction de la LED. Le pic secondaire est maximum vers $550 \mathrm{~nm}$ et représente l'émission lumineuse des luminophores excités par la lumière bleve (fluorescence). La combinaison (synthèse additive) de ces deux composantes est à l'origine de la perception d'une couleur blanche par l'œil. La courbe rouge est la courbe de photo-toxicité rétinienne de la lumière bleue (spectre d'action) représentée par la fonction $B(\lambda)$. Cette fonction est maximale entre 435 et $440 \mathrm{~nm}$, dans le domaine spectral correspondant au pic blev émis par les LED blanches.

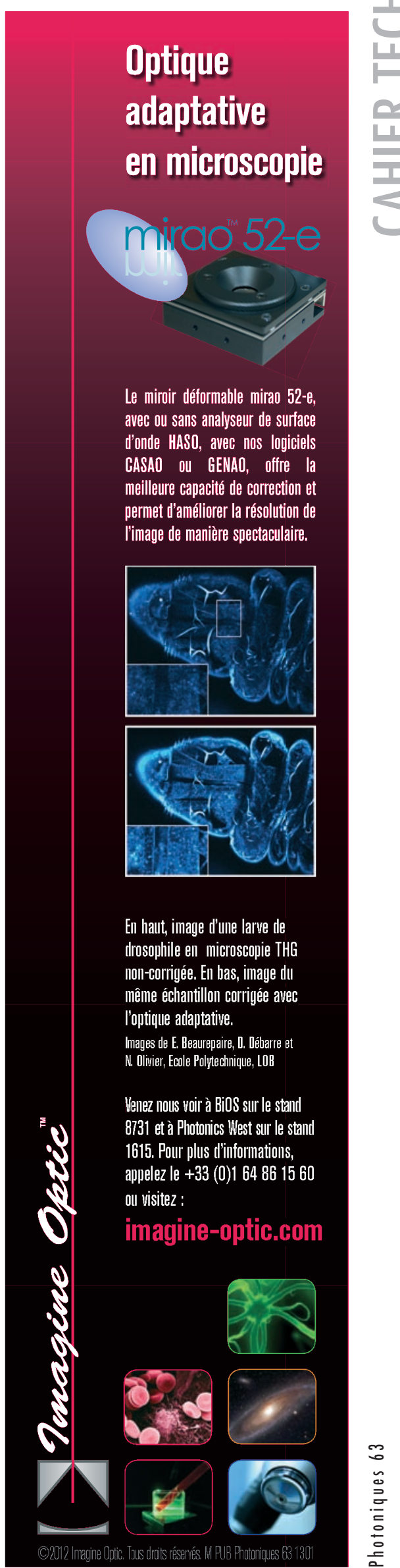

www. photoniques.com 
assez peu sensible aux longueurs d'onde de son spectre [4].

En excluant les rayonnements ultraviolets, généralement filtrés par les milieux oculaires situés en avant de la rétine, les lésions photochimiques rétiniennes ne sont provoquées que par les photons situés dans les zones bleues et violettes du spectre lumineux [5]. Ces photons sont responsables de processus oxydatifs dans l'épithélium pigmentaire rétinien (EPR) [4] [5] [6]. Situé sous la rétine, I'EPR assure le renouvellement des pigments visuels et l'élimination quotidienne d'une partie de la zone photosensible des photorécepteurs. Cette élimination du segment externe des photorécepteurs conduit à l'accumulation de dépôts photo-sensibilisateurs à la lumière bleue, la lipofuscine. Lorsque ce composé est excité par des photons de courtes longueurs d'onde, il génère des espèces réactives d'oxygène, comme les radicaux libres par exemple, qui sont responsables de lésions qui peuvent être irréversibles et conduire à la mort des neurones visuels et donc altérer la vision. Ces phénomènes de stress oxydatifs sont observés également dans plusieurs pathologies, dont la DMLA (dégénérescence maculaire liée à l'âge), ainsi que dans les processus de vieillissement. À l'inverse des lésions thermiques, les lésions photochimiques dépendent de l'éclairement cumulé auquel la rétine est exposée [7]. Celui-ci peut être le résultat d'une exposition courte à une source intense mais aussi d'une exposition de faible niveau mais répétée (exposition de type chronique).

Les niveaux d'éclairement rétinien produits par les LED basées sur les technologies actuelles sont trop faibles pour provoquer des lésions thermiques. Par contre, les LED peuvent potentiellement provoquer de forts niveaux d'éclairement rétinien dans le bleu, ce qui motive de connaître les niveaux atteints.

L'exposition rétinienne à la lumière bleue peut être estimée en suivant les recommandations de I'ICNIRP (International Commission on Non-lonising Radiation Protection) [4]. Pour la population générale, le spectre d'action de la lumière bleue sur la rétine est représenté par la fonction $B(\lambda)$ dont les valeurs sont précisément tabulées dans [4]. Cette fonction atteint son maximum entre 435 et $440 \mathrm{~nm}$ (figure 2). La fonction $B(\lambda)$ peut être utilisée pour intégrer la luminance spectrique $L_{\lambda}$ d'une source d'éclairage, mesurée dans un champ de vision effectif qui dépend du temps d'exposition considéré :

$$
L_{B}=\int L_{\lambda} B(\lambda) d \lambda
$$

On obtient alors la quantité $L_{B^{\prime}}$ appelée la luminance pondérée dans le bleu. Une valeur limite d'exposition (VLE) a été fixée par l'ICNIRP pour la luminance pondérée $L_{B}$ selon le temps d'exposition considéré : $L_{B}<10^{6} / t$

avec $L_{B}$ exprimée en $\mathrm{Wm}^{-2} \mathrm{sr}^{-1}$ et $t$ exprimé en $\mathrm{s}$.

Durant les trois dernières années, les niveaux d'exposition à la lumière bleue rétinienne produits par les LED ont été fournis en termes de luminance pondérée $L_{B}$ par des fabricants de LED et des syndicats professionnels d'éclairage mais aussi par des laboratoires indépendants et des agences publiques. Ces données montrent que les niveaux produits à la distance de 200 mm de l'œil par certaines LED dépassent les VLE au-delà d'une durée d'exposition de l'ordre de quelques secondes pour des LED bleues et de quelques dizaines de secondes pour des LED de type blanc froid (tableau 2).

Par conséquent, la toxicité potentielle de certaines LED observées à courte distance ne peut pas être négligée. Cependant, lorsque la distance d'observation est portée à $1 \mathrm{~m}$, les durées limites d'exposition (temps au-delà duquel les VLE sont dépassées) augmentent très rapidement à quelques milliers ou quelques dizaines de milliers de secondes. Ces temps très longs fournissent une marge de sécurité raisonnable pour affirmer que les LED, prises individuellement, ne présentent pas de risque pour la rétine si elles sont

\begin{tabular}{|c|c|c|c|c|}
\hline Type de LED & $\begin{array}{l}\text { Flux énergétique (W) } \\
\text { ou lumineux (Im) }\end{array}$ & $\begin{array}{l}\text { Luminance énergétique } \\
\left(\mathrm{Wm}^{-2} \mathrm{sr}^{-1}\right) \\
\text { ou lumineuse }\left(\mathrm{cd} / \mathrm{m}^{2}\right)\end{array}$ & $\begin{array}{l}\text { Durée d'observation à partir } \\
\text { de laquelle les valeurs limites } \\
\text { d'exposition (VLE) de la rétine } \\
\text { à la lumière bleue sont dépassées } \\
\text { (valeurs données pour une distance } \\
\text { d'observation de } 200 \mathrm{~mm} \text { ) }\end{array}$ & $\begin{array}{c}\text { Groupe de risque } \\
\text { selon la norme } \\
\text { NF EN } 62471\end{array}$ \\
\hline \multirow{3}{*}{ Bleu } & $0,07 \mathrm{~W}$ & $21000 \mathrm{Wm}^{-2} \mathrm{sr}^{-1}$ & $100 \mathrm{~s}$ à $10000 \mathrm{~s}$ & RG 1 : risque faible \\
\hline & $0,5 \mathrm{~W}$ & $150000 \mathrm{Wm}^{-2} \mathrm{sr}^{-1}$ & 15 d̀ $20 \mathrm{~s}$ & RG 2 : risque modéré \\
\hline & $1 \mathrm{~W}$ & $300000 \mathrm{Wm}^{-2} \mathrm{sr}^{-1}$ & 3 à $4 \mathrm{~s}$ & RG 2 : risque modéré \\
\hline \multirow{2}{*}{ Blanc froid } & $100 \mathrm{~lm}$ & $1,6.10^{7} \mathrm{~cd} / \mathrm{m}^{2}$ & VLE non atteinte & RG 0 : sans risque \\
\hline & $200 \mathrm{~lm}$ & $3,2.10^{7} \mathrm{~cd} / \mathrm{m}^{2}$ & 50 à $100 \mathrm{~s}$ & RG 2 : risque modéré \\
\hline \multirow{2}{*}{ Blanc neutre } & $100 \mathrm{~lm}$ & $1,6.10^{7} \mathrm{~cd} / \mathrm{m}^{2}$ & VLE non atteinte & RG 0 : sans risque \\
\hline & $200 \mathrm{~lm}$ & $3,2 \cdot 10^{7} \mathrm{~cd} / \mathrm{m}^{2}$ & 100 s à $10000 \mathrm{~s}$ & RG 1 : risque faible \\
\hline \multirow{2}{*}{ Blanc chaud } & $100 \mathrm{~lm}$ & $1,1.10^{7} \mathrm{~cd} / \mathrm{m}^{2}$ & \multirow{2}{*}{ VLE non atteinte } & \multirow{2}{*}{ RG 0 : sans risque } \\
\hline & $200 \mathrm{~lm}$ & $2,2.10^{7} \mathrm{~cd} / \mathrm{m}^{2}$ & & \\
\hline
\end{tabular}

Tableau 2. Tableau tiré de la référence [2] : examen des valeurs limites d'exposition de la rétine à la lumière bleve émise par de plusieurs LED blanches et bleue à la distance de $200 \mathrm{~mm}$ de l'œil. Les intervalles de valeurs correspondent aux variations des résultats observés avec plusieurs types de spectres et de longueur d'onde dominante. 
observées au-delà d'un mètre par un individu n'appartenant pas à une population sensible.

À l'inverse, certains usages des LED présentent potentiellement un risque rétinien. Des exemples sont donnés ici, sans prétendre à l'exhaustivité :

- Tests et ajustements de LED par les ingénieurs, opérateurs et installateurs lors de la conception, la fabrication et l'installation de ces dispositifs. Les risques sont accrus par l'utilisation de systèmes optiques grossissants (collimateurs) qui augmentent optiquement la taille de la source et conduisent à une augmentation de la luminance pondérée $L_{B}$.

- Jouets incorporant des LED. Les risques sont ici liés à la plus grande transparence du cristallin des enfants, ce qui les place de facto dans les populations sensibles pour lesquels I'ICNIRP préconise d'utiliser un autre spectre d'action dont l'amplitude est dix fois plus grande que celle de la fonction $B(\lambda)$. Un facteur aggravant est que les LED sont souvent regardées par les enfants à des distances extrêmement courtes.

- Les LED utilisées dans les phares automobiles de jour, qui présentent actuellement les plus hauts niveaux de luminance pondérée $L_{B}$ parmi toutes les LED d'éclairage. Le faisceau de ces dispositifs peut être dirigé dans le champ de vision de bébés et d'enfants situés à proximité des véhicules et à la même hauteur que les phares.

- Certains types de lampes directionnelles à LED pour applications domestiques, comportant des LED bleues ou blanches froides associées à des collimateurs optiques. Possédant des niveaux de luminance pondérée élevés, elles peuvent être installées à proximité des yeux des utilisateurs (luminaire d'appoint pour la lecture, lampe de chevet, etc.).

Les conclusions tirées pour des composants LED ne peuvent pas être appliquées à tous les dispositifs utilisant des LED. En effet, la luminance pondérée d'un système doit être évaluée indépendamment de celle de ses composants. Dans le cas de lampes ou de luminaires d'éclairage, la valeur de $L_{B}$ est en effet très différente. Par exemple, un luminaire utilisant des LED à forte luminance peut avoir une luminance faible avec la simple utilisation d'un diffu-

\begin{tabular}{l|l}
\hline Groupe de risque RG 0 (absence de risque) & $t_{\text {lim }} \geq 10000 \mathrm{~s}$ \\
\hline Groupe de risque RG 1 (risque faible) & $100 \mathrm{~s} \leq t_{\lim }<10000 \mathrm{~s}$ \\
\hline Groupe de risque RG 2 (risque modéré) & $0,25 \mathrm{~s} \leq t_{\lim }<100 \mathrm{~s}$ \\
\hline Groupe de risque RG 3 (risque élevé) & $t_{\text {lim }}<0,25 \mathrm{~s}$
\end{tabular}

Tableav 3. Définition des groupes de risque permettant de classer les sources lumineuses à LED selon le risque rétinien dû à la lumière bleue (norme NF EN 62471).

seur optique. À l'inverse, une lampe composée d'un assemblage dense de LED à faible luminance peut avoir une forte valeur de $L_{B}$ et présenter plus de risque potentiel.

Par ailleurs, l'exposition simultanée à plusieurs lampes ou luminaires à LED augmente l'éclairement rétinien et nécessite donc une évaluation au niveau de l'installation complète d'éclairage, comme par exemple une installation d'éclairage intérieur complexe ou d'éclairage scénographique [8].

Pour toutes les LED et les dispositifs incorporant des LED, une évaluation du risque photobiologique doit être effectuée pour déterminer si les VLE peuvent être dépassées dans les conditions d'utilisation. Cette évaluation de risque est proposée par un nombre croissant de laboratoires d'essais spécialisés dans la photométrie des sources lumineuses. En France, le LNE (Laboratoire national de métrologie et d'essais), le CSTB (Centre scientifique et technique du bâtiment) et I'INRS (Institut de recherche et de sécurité) effectuent régulièrement ce type d'évaluation.

La méthodologie expérimentale utilisée par les laboratoires pour l'évaluation du risque photobiologique des sources lumineuses est la publication CIE S009 [9], publication dont le contenu a été transposé dans la norme internationale IEC 62471 et adapté en Europe puis en France dans la norme NF EN 62471 [10].

\section{La méthodologie d'évaluation du risque dans la norme de sécurité photobiologique NF EN 62471}

La norme NF EN 62471 porte sur la sécurité photobiologique des lampes et des appareils utilisant des lampes. Elle propose une classification d'une source lumineuse selon plusieurs groupes de risque (RG). La norme prend en compte l'ensemble des risques photobiologiques relatifs aux impacts sur la peau et les yeux dans les domaines visible, infrarouge et ultraviolet.

Quatre groupes de risque sont définis : RG 0 (absence de risque), RG 1 (risque faible), RG 2 (risque modéré), RG 3 (risque élevé). Le groupe de risque dépend de la durée limite d'exposition $t_{\text {limm }^{\prime}}$ qui est la durée au-delà de laquelle les VLE sont dépassées. Si on ne considère, dans le cas des LED, que le risque rétinien dû à la lumière bleue, la définition des groupes de risque est décrite dans le tableau 3.

Notons que le groupe de risque dépend de la luminance pondérée $L_{B}$. II est donc fonction de la distance d'observation au travers du champ de vision effectif utilisé pour l'évaluation de la luminance. La norme NF EN 62471 introduit deux critères différents pour déterminer la distance d'observation à laquelle le groupe de risque doit être évalué :

- Les sources d'éclairage général doivent être évaluées à la distance correspondant à un éclairement de $500 \mathrm{~lx}$.

- Les autres types de sources doivent être évalués à une distance de $200 \mathrm{~mm}$.

Pour les LED, prises en tant que composants, il n'y a pas d'ambiguïté dans le choix de la distance d'évaluation puisque les LED seules ne constituent pas des produits finis d'éclairage général. La norme NF EN 62471 requiert donc une évaluation du groupe de risque à la distance de 200 $\mathrm{mm}$. Ainsi, selon cette norme, on constate que certaines LED bleues et blanc froid sont dans la catégorie RG 2 (tableau 2) qui correspond à un risque modéré.

Le choix de la distance d'évaluation de risque dans la norme NF EN 62471 est parfois ambigu et ne représente pas l'usage réel des produits. Par exemple, dans 
l'éclairage scénographique, les artistes sont exposés à des éclairements pouvant largement dépasser les 500 Ix. Dans ce cas, I'application de cette norme sous-estime largement les risques photobiologiques. Dans une situation plus courante, les lampes directionnelles utilisées dans les pièces $d$ 'habitation produisent un éclairement de $500 \mathrm{Ix}$ à une distance de quelques mètres, alors qu'elles peuvent être accessibles à des distances beaucoup plus courtes. À l'opposé, dans le domaine de l'éclairage public, les luminaires produisent des niveaux d'éclairement de quelques dizaines de Ix, ce qui est inférieur au niveau de $500 \mathrm{~lx}$ requis dans la norme. Dans ce cas, le risque photobiologique est surestimé.

II est intéressant de noter que la stricte application de la norme NF EN 62471 aux lampes et luminaires à LED à usage domestique fournit des groupes de risque RG 0 et RG 1, similaires aux groupes de risques des lampes traditionnelles à incandescence ou fluorescentes. Néanmoins, lorsque le critère de distance de $200 \mathrm{~mm}$ est choisi, des campagnes de mesures $[2,6]$ ont mis en évidence quelques produits d'éclairage LED dont le groupe était RG 2. Ces résultats démontrent que la technologie LED augmente potentiellement l'exposition rétinienne à la lumière bleue dans I'habitat, ce qui soulève des interrogations sur l'impact généré sur les populations sensibles comme les enfants. À l'heure de la rédaction de cet article, le public n'est pas informé des risques potentiels puisque aucun marquage de sécurité photobiologique n'est présent sur les produits ou leur emballage.

Pour les produits d'éclairages installés par des professionnels (électriciens, installateurs, éclairagistes), la notion de distance minimale de sécurité est une grandeur qui pourrait être communiquée par les fabricants de manière à ce que les produits soient posés dans de bonnes conditions. Cette distance est par définition, la distance d'observation pour laquelle le groupe de risque ne dépasse pas RG 0 .

Il est important de rappeler que les autres technologies de lampes d'usage professionnel, comme les lampes halogènes de forte puissance et les lampes à décharge de haute intensité (HID) ont des groupes de risques RG 2. À l'inverse des LED, ce sont les émissions dans l'ultraviolet et l'infrarouge qui sont les plus critiques [3]. Les impacts sur la peau sont potentiellement plus élevés avec ce type de lampe qu'avec les LED.

\section{Limitations de la norme NF EN 62471 concernant les populations sensibles}

L'ICNIRP précise dans ses recommandations [4] que les valeurs limites d'exposition définies pour la population générale, et reprises dans la norme NF EN 62471 , ne sont pas applicables pour les expositions chroniques et répétées à la lumière bleue. En particulier, ces valeurs ne prennent pas en compte les expositions répétées dites "sous-critiques", inférieures aux VLE, qui se cumulent au cours de très longues périodes, voire au cours de la vie entière.

La norme NF EN 62471 ne considère pas non plus la sensibilité accrue aux rayonnements optiques pour certaines populations sensibles. Ces populations sont les suivantes :

- personnes ayant une affection préexistante des yeux ou de la peau et pour lesquels la lumière artificielle déclenche ou aggrave certains symptômes ;

- personnes aphakes (personnes dont l'œil ne possède pas de cristallin) et pseudophakes (personnes possédant un cristallin artificiel, souvent après une opération de la cataracte) qui filtrent insuffisamment les rayonnements de courte longueur d'onde (UV, violet, bleu) ;

- les enfants, dont le cristallin est naturellement beaucoup plus transparent que celui des adultes;

- les personnes âgées dont la rétine est plus sensible aux rayonnements optiques de courte longueur d'onde en raison de l'accumulation au cours de la vie de dépôts photo-sensibilisants dans la rétine.

La norme de sécurité photobiologique relative aux LED et aux systèmes d'éclairage en général, devrait donc être adaptée à ces populations, en prenant notamment en compte la courbe de phototoxicité (spectre d'action) de la lumière bleue donnée par I'ICNIRP pour les populations sensibles.
En plus des lésions de type photochimique de la rétine résultant d'une exposition aiguë à la lumière bleue, il subsiste une méconnaissance des effets des expositions chroniques et répétées à faible dose. Ces effets sont actuellement en cours d'étude par des ophtalmologistes, des biologistes et des physiciens spécialisés en optique. En France, le projet RETINALED cherche à comprendre les effets de la lumière émise par les LED sur la rétine de rongeurs exposés sur de longues durées à des éclairements relativement faibles mais permanents. En attendant les résultats de ces recherches, le principe de précaution voudrait que des mesures de protection individuelles soient mises en place chez les travailleurs particulièrement exposés lors de leurs activités professionnelles et chez les populations sensibles décrites ci-dessus.

\section{En conclusion}

Grâce à leurs propriétés uniques d'émission lumineuse, les LED sont sur le point de devenir les sources de lumière les plus utilisées dans le monde pour les applications d'éclairage. Les risques oculaires présentés par ces nouvelles sources sont malheureusement liés de manière intrinsèque à ces propriétés : un flux lumineux important émis par une très petite surface, conduisant à de fortes luminances, ainsi qu'un spectre comportant une composante bleue très marquée. La combinaison de ces deux caractéristiques augmente le risque potentiel de lésion photochimique de la rétine, en comparaison avec des sources traditionnelles de lumière telles que les lampes à incandescence et fluorescentes.

Les industriels dominant le marché de l'éclairage mesurent parfaitement les niveaux de risque photobiologique des produits à LED qu'ils fabriquent. Beaucoup de dispositifs sont maintenant orientés vers l'émission de lumière blanche de type blanc chaud, réduisant ainsi la composante bleue du spectre. Ils intègrent également des diffuseurs de lumière ou d'autres systèmes optiques visant à réduire les niveaux de luminance. Parmi les produits d'éclairage à LED qui ont été testés lors 
de plusieurs campagnes de mesures, la grande majorité présentait des groupes de risque nul ou faible pour la population générale lorsque la distance d'observation était fixée à $200 \mathrm{~mm}$. Cependant, il subsiste sur le marché des lampes et des luminaires dont le groupe de risque est plus élevé que celui des produits traditionnels. À I'heure actuelle, aucune mention n'est faite du groupe de risque ou de la distance minimale de sécurité sur ces produits. II est donc impossible pour le public d'identifier ces produits.

L'évaluation du risque pour la rétine lié à l'émission lumineuse des LED peut s'effectuer en laboratoire sur la base de la norme NF EN 62471 qui n'est pas parfaitement claire en ce qui concerne le choix de la distance d'observation à considérer. De plus, cette norme ne prend pas en compte les populations sensibles que sont les enfants, les aphakes et pseudophakes ainsi que les personnes âgées, en dépit du fait que ces populations sont plus sensibles à l'exposition aux lumières bleues et violettes.

Au-delà de l'évaluation d'un système d'éclairage effectué en laboratoire sur la base de la norme NF EN 62471, il subsiste une incertitude quant aux niveaux réels d'exposition de la rétine à la lumière bleue émise par un ensemble de lampes ou de luminaires à LED dans des installations d'éclairage au sein desquelles l'œil est soumis à une multiplicité de sources présentes dans son champ de vision. Seules des études menées sur des installations réelles permettront de statuer sur l'innocuité ou la toxicité potentielle de ces installations.

La connaissance des mécanismes de la toxicité rétinienne de la lumière bleue est encore incomplète. En particulier, les effets des expositions chroniques et de leur accumulation pendant de longues périodes sont encore à étudier et font l'objet de nombreuses recherches dans le monde entier. En ce qui concerne les LED, la meilleure compréhension de ces effets à long terme sur la rétine est fondamentale si l'on veut que cette véritable révolution dans le domaine de l'éclairage ne vienne pas compromette notre sécurité et notre santé.

\section{Références}

[1] Tähkämo L., Bazzana M., Ravel P., Grannec F., Martinsons C., Zissis G. "Life Cycle Assessment of Light Emitting Diode Downlight Luminaire a case study", accepted for publication in The International Journal of Life Cycle Assessment

[2] «Effets sanitaires des systèmes d'éclairage utilisant des diodes électroluminescentes (LED) », Saisine ${ }^{\circ} 2008-S A-0408$, Rapport d'expertise collective de l'Agence Nationale de Sécurité Sanitaire de l'Alimentation, de l'Environnement et du Travail (ANSES), octobre 2010, téléchargeable sur le site internet de l'ANSES : www.anses.fr

[3] "Health Effects of Artificial Light", Opinion of the Scientific Committee on Emerging and Newly Identified Health Risks (SCENIHR), European Commission, March 2012, ISSN 1831-4783, téléchargeable : http://ec.europa.eu/health/ scientific_committees/policy/index_en.htm

[4] International Commission on Non-lonizing Radiation Protection, "Guidelines on limits of exposure to broad-band incoherent optical radiation $(0.38 \text { to } 3 \mu \mathrm{m})^{\prime \prime}$, Health Physics $73(3)$ (1997), 539-554

[5] Noell W.K., Walker V.S., Kang B.S., Berman S. "Retinal damage by light in rats", Invest. Ophthalmol. 5(5) (1966), 450-473

[6] Behar-Cohen F., Martinsons C., Viénot F., Zissis G., Barlier-Salsi A., Cesarini J.P., Enouf O., Garcia M., Picaud S., Attia D. "Light-emitting diodes (LED) for domestic lighting: Any risks for the eye?", Progress in Retinal and Eye Research 30(4) (2011), 239-257

[7] Ham W.T., Mueller H.A., Sliney D.H. "Retinal sensitivity to damage from short wavelength light", Nature 260 (1976), 153-155.

[8] Barlier-Salsi A., Salsi S., « Mesures des rayonnements optiques aux postes de travail comparaison de différentes méthodes et matériels de mesure », Radioprotection 45(3), 307-320

[9] Photobiological safety of lamps and lamp systems, publication CIE S009 (2002) de la Commission Internationale de l'Éclairage

[10] Sécurité photobiologique des lampes et des appareils utilisant des lampes, norme NF EN 62471 (2008), publiée par I'AFNOR

Christophe Martinsons est responsable de la division Éclairage et électromagnétisme au sein de la direction Santé et confort du Centre scientifique et technique du bâtiment (CSTB) situé à Saint-Martin-d'Hères.

Christophe Martinsons est également expert à l'Agence de sécurité sanitaire de l'alimentation, de l'environnement et du travail (ANSES). II a notamment contribué à l'élaboration du rapport d'expertise de l'ANSES sur les impacts sanitaires des LED [2].

\section{Micro positionnement pour l'usinage laser} Les contrôleurs, les platines et les systèmes
Aerotech augmentent la précision et le rendement de votre application tout en maximisant les temps de bon fonctionnement. PRO Series

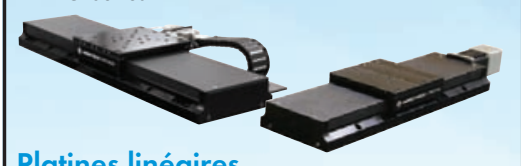

Platines linéaires

- Courses de 50 à $1500 \mathrm{~mm}$

- Vitesse jusqu'à $2 \mathrm{~m} / \mathrm{s}$

- Conçus avec des joints latéraux et un capot de protection en tôle

- Entrainement à vis à bille ou à moteur linéaire

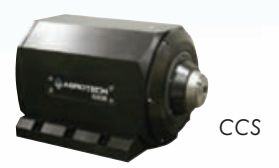

Rotation à entrainement direct

- Mandrin à serrage et desserrage pneumatique

- Collet captif minimise les défauts axiaux pendant le serrage/desserrage

- Ouverture central pour alimentation

- Moteur brushless et codeur dans I'axe

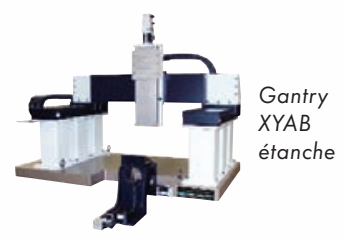

Systèmes Gantry

à moteurs linéaires

- Vitesse de $3 \mathrm{~m} / \mathrm{s}$ et accélération de $5 \mathrm{~g}$

- Précision exceptionnelle, haut rendement et grande productivité

- Versions étanches et options customisées pour votre application

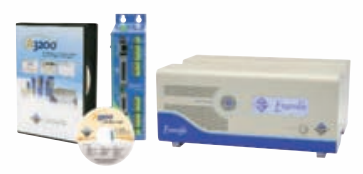

Plateformes contrôleurs avancés

- Mono et multiaxes

- Fonctions de contrôle avancées et software modulaire

- Environnement d'automation embarqué PLC

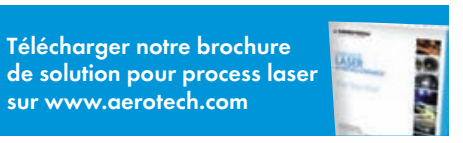

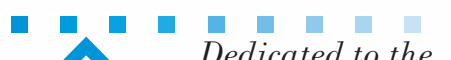

Dedicated to the
Science of Motion
Aerotech France, BP 70043
45702 Villemandeur Cedex
Ph: +33 238970830
Email: ventes@aerotech.com

www.aerotech.com 